\title{
The research for the receiving technique of iterative joint
}

\author{
JingPeng $\mathrm{GaO}^{1, \mathrm{a}}$, LiHua Yang ${ }^{2, \mathrm{~b}}$ and ChaoQun $\mathrm{Wu}^{3, \mathrm{c}}$ \\ ${ }^{12}$ College of Information and Communication Engineering \\ Harbin Engineering University, Harbin, 150001, China \\ ${ }^{3}$ School of Automobile and Traffic Engineering \\ Heilongjiang Institute of Technology, Harbin, 150001, China \\ agaojingpeng@hrbeu.edu.cn, byanglihua@hrbeu.edu.cn, cojpmcu@126.com
}

\begin{abstract}
Keywords: Soft Interference Cancellation Detection Algorithm; Improved Maximum a Posteriori Algorithm; Iterative Joint Receiving Technique.

Abstract. Aiming at the problem that in traditional MIMO-OFDM receiver the channel decoding, channel estimation and signal detection are independent and the error rate is relatively high, this paper proposes an iterative joint receiving scheme, improve it, and gets a modified iterative joint receiving technique scheme. The theoretical research and the simulation result indicated: compared with the iterative joint receiving scheme, in the same signal to noise ratio, the performance of the modified iterative joint receiving technique scheme has improved about one order of magnitude higher. In the case of same error rate, the new scheme also has improved more than $2 \mathrm{~dB}$. So the modified iterative joint receiving technique scheme can improve the performance of the MIMO-OFDM system effectively.
\end{abstract}

\section{Introduction}

MIMO-OFDM technique has become one of the potential solutions in physical layer that can achieve high speed data transmission in the wireless channel. It has vast research and developing space. Channel decoding, channel estimation and signal detection are the key technologies of the MIMO-OFDM system, and the three technologies work independently in the system. Aiming at the channel estimation and signal detection, the paper [1] proposed an algorithm to improve system performance. The article [2] researched on the signal detection and the channel decoding to improve system performance.Turbo code came up with by C.Berrou in the 1993. It has become the research hotspot for the vast scholars, since its performance approaches to the Shannon theoretical limits and iterative decoding algorithm is novel [3]. Turbo interation theory as a new effective technology to improve system performance has been widely used in the channel equalization, channel decoder and joint iteration dispose.

This paper contactes channel estimation, signal detection and channel decoding by the Turbo iteration theory to make the three do interactive iteration process by soft information. By this way, improve channel estimation performance, make the signal detection data more precision and improve the whole system performance.

\section{Iterative Joint Receiving Technique}

In the system receiver, we get the original channel state information by dispatching optimal training sequence. Then use the original channel state information for channel decoding and signal detection. After the decoder produces the soft information, transform it to symbo estimation information through feedback information processing unit by the principal of Turbo Iterative. And symbol estimation information as the virtual training sequence feedback to the channal estimator correctes the original channel state information. At the same time, put the corrected channel state information into the signal detector. And use the prior probability which is feedbacked by the signal detector to promote the signal detection performance. Send the soft information which is output by detector to the decoder for channel decoding. The channal estimator, signal detector and channal decoder are 
processed more than once by the principal of iterative joint. Finally, the soft informaton is output by hard decision, and make the updated channel state information as the next signal channel estimation starter for the next signal with iterative joint receiving.

\section{The Modified MAP Arithmetic}

In the MIMO-OFDM iterative joint receiving system, the common soft input and soft output signal detection algorithm are MAP arithmetic[4] and MMSE-SIC arithmetic. The MMSE-SIC arithmetic is a typical soft input and soft output signal detection arithmetic.It has better detection property and the lower complexity.So it is widely used. In the iterative joint receiving system, the MMSE-SIC arithmetic still has the high complexity. We can simplify the MAP signal detection arithmetic, using the max-log approximate:

$$
\log \left(e^{x_{1}}+e^{x_{2}}+\cdots+e^{x_{n}}\right) \approx \max _{i} x_{i} .
$$

The posterior logarithm likelihood ratio can be approximated as:

$$
\begin{aligned}
\operatorname{LLR}\left(b_{l, i} \mid \mathbf{y}\right) & =\ln \frac{p\left(b_{l, i}=1 \mid \mathbf{y}\right)}{p\left(b_{l, i}=0 \mid \mathbf{y}\right)} \\
& \approx \min _{x \in S_{l, i}^{-}}\left(\frac{1}{2 \sigma_{z}^{2}}\|\mathbf{y}-\mathbf{H x}\|^{2}\right)-\min _{x \in S_{l, i}^{+}}\left(\frac{1}{2 \sigma_{z}^{2}}\|\mathbf{y}-\mathbf{H x}\|^{2}\right) \\
& =\min _{x \in S_{l, i}^{\perp}} D(x)-\min _{x \in S_{l, i}^{+}} D(x)
\end{aligned}
$$

Among them, $D(x)=\frac{1}{2 \sigma_{z}^{2}}\|\mathbf{y}-\mathbf{H x}\|^{2}$.

When the number of transmitting antenna and the modulation order increase, the computation complexity will increase. In order to reduce the complexity of the detecting algorithm, according to the modified sphere decoding detecting algorithm[7], use the formula $C C_{0}^{2}=\left\|\mathbf{r}-\mathbf{\Omega} \mathbf{s}_{M M S E}\right\|^{2}$ to reduce the candidate vector set. Let B the reduced candidate vector set, and the formula (2) can be approximated as:

$$
\operatorname{LLR}\left(b_{l, i} \mid \mathbf{y}\right) \approx \min _{x \in S_{l, i, B}} D(x)-\min _{x \in S_{l, i, B}^{+}} D(x)
$$

Among them, $S_{l, i, B}^{-}=S_{l, i}^{-} \cap B, S_{l, i, B}^{+}=S_{l, i}^{+} \cap B$.

Because the candidate vector set is reduced, it may exist the problem that the candidate vector set $S_{l, i}^{+}$or $S_{l, i}^{-}$is empty. The formula(3) is not reliable any more. In order to avoid this case, definition

$$
\begin{aligned}
& \mathbf{x}_{B}=\underset{x \in B}{\arg \min }\left(\frac{1}{2 \sigma_{z}^{2}}\|\mathbf{y}-\mathbf{H x}\|^{2}\right) \\
& \text { If } S_{l, i, B}^{-}=S_{l, i}^{-} \cap B=\varphi \text {, set } \\
& \min _{x \in S_{l, i, B}^{-}} D(x)=\frac{1}{2 \sigma_{z}^{2}}\left\|\mathbf{y}-\mathbf{H} \mathbf{x}_{B}\right\|^{2} \\
& \text { If } S_{l, i, B}^{+}=S_{l, i}^{+} \cap B=\varphi \text {, set } \\
& \min _{x \in S_{l, i, B}^{-}} D(x)=\frac{1}{2 \sigma_{z}^{2}}\left\|\mathbf{y}-\mathbf{H} \mathbf{x}_{B}\right\|^{2}
\end{aligned}
$$


Because the candidate vector set is reduced, it may appear the condition that the real check point is not in B, the formula(5) and formula (6) may exist the problem that the calculated amount is too large. In order to guarantee the reliability of detecting algorithm, designe a threshod value $\Xi$ to avoid higher loss of performance, the formula (5)and formula (6) can be updated to:

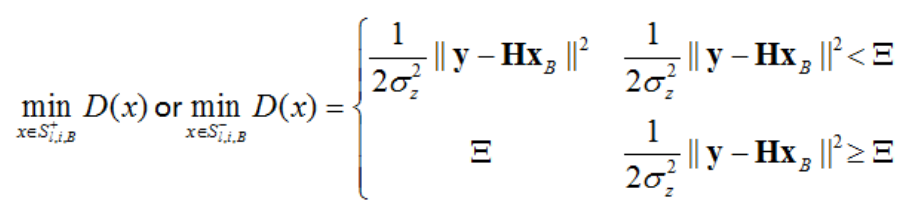

Among them, threshod value $\Xi$ is 2 generaly. According to the formula(3), IMAP arithmetic can only involve a little multiplication and comparison, It reduces the complexity detecting algorithm by sacrificing MAP performance detection.

\section{The Modified Receiving Technique of Iterative Joint}

For achieve the modified receiving technique of iterative joint. Firstly, the technique fetchs information from OFDM mediate information. Acquire the channal beginning estimation by extracted optimal training sequence through time domain channal estimation. Secondly, the technique uses the time domain channal estimation arithmetic based on SAGE to revise channal state information and do the signal soft detection combining OFDM data information with modified IMAP arithmetic. Then, acquire the soft decode information by de-interleaver and MAX-Log-MAP decoder to finish the iterative processing. If the result is not satisfied to the output condition of iterative joint, put the interlaced soft decode information into IMAP signal detector for detection the soft information and iterative update unitl satisfy the iterative joint output condition. Later, put the output soft decode information by hard decision to finish the operation of the current OFDM signal. Finally, put the data information by soft symlinks estiator and extractive OFDM into SAGE time domain channal estimator to update the next sigal channal state information. By the signal detector and the channal decoder, process the next signal information until the whole frame data decode finished.

\section{Simulation Result and Analysis}

Simulated the performance of the modified terative joint receiving technique referred in this papaer, the simulation parameters are: count of FFT is 128 , channal model is suburban macro community, the number of transmitting antenna is 3 , carrier frequency is $2.4 \mathrm{GHz}$, channal bandwidth is $5 \mathrm{MHz}$, cyclic prefix number is 32, the number of receving antenna is 3, the structure of frame is 10 OFDM symbols, signal detection arithmetic is IMAP arithmetic, channal estimation is MMSE-SIC arithmetic, MMSE-SIC arithmetic iterations is 3. Assumes that the transmitting antenna and receiving antenna are independent of each other, and compare the normalization error of mean square with the bit error rate in modified receiving technique of iterative joint.

Figure 1 shows the changing curves of the normalization error of mean square with the change of Turbo iterative times in the modified receiving technique of iterative joint. Among them, the modulation is 8QAM modulation. Simulation result shows that with the signal to noise ratio increases, the normalization error of mean square of the modified receiving technique of iterative joint will decrease accordingly. And with the iterations increasing, the normalization error of mean square is not improved obviously. In order to decrease the system complexity, the modified receiving technique of iterative joint only does the estimation and the correction of channal state information when the next OFDM symbol is dealing in the case of the modified receiving technique of iterative joint. So the change of the Turbo iterations can not improve the performance of channal estimation obviously in the modified receiving technique of iterative joint.

Figure 2 shows the changing curves of the bit error rate with the change of Turbo iterative times in the modified receiving technique of iterative joint. Among them, the modulation is 8QAM 
modulation. Simulation result shows that the bit error rate of the modified iterative joint receiving technique will decrease with the increased signal to noise ratio. With the iterations increasing, the bit error rate can be improved. The performance of the former three times iteration improves obviously, and after the three times iteration, the performance mainly convergent. As the iterations increases, the useful information provided by the channal decoding will increase accordingly, meanwhile this way will transmit the error of the channal decoding to signal detector. So, the performance can improve obviously, but the arithmetic will keep convergence mainly after three times iteration. Considering the reliability and the validity of the system comprehensively, let the iteration be 3 .

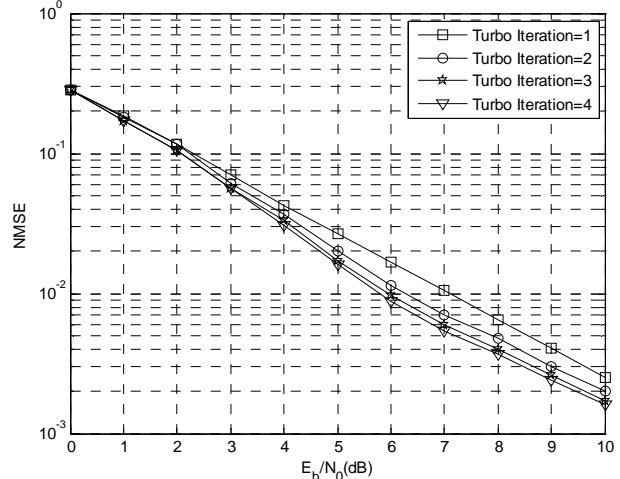

Figure 1: The curve of normalized mean square error

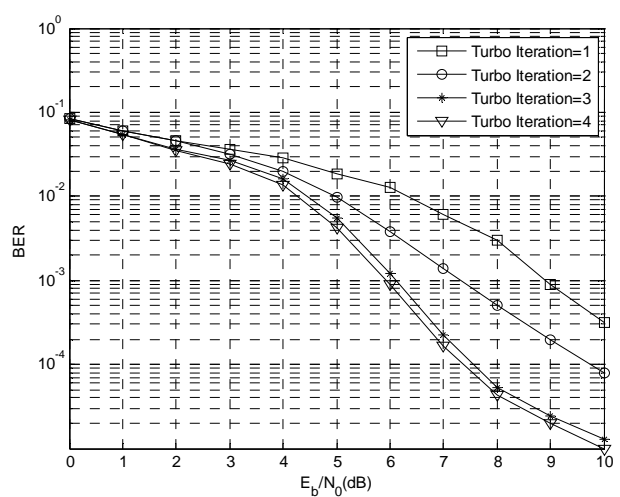

Figure 2: The curve of bit error rate

\section{Summary}

This paper puts forward a modified receiving designing scheme of iterative joint aiming at the problem that in traditional MIMO-OFDM system, the channel decoding, channel estimation and signal detection work independently, and much information loss because of hard information. The quality of the signal detection is promoted, the output error rate of channal decoding is reduced, and the performance of the system Introduce is promoted by means of introducing the channal decoding into the receiving of the iterative joint to realize the combine with channel decoding, channel estimation and signal detection.It has effective practical engineering value.

\section{Acknowledgements}

This work was financially supported by the Fundamental Research Funds for the Central Universities of China (No.HEUCF150814). The authors would like to thank the paper editor and the reviewers for their valuable comments and suggestions.

\section{References}

[1] R. P. Salvo, and R. R. Müller, in: Joint twofold-iterative channel estimation and multiuser detection for MIMO-OFDM systems. edited by Wireless Communications, IEEE chapter, 11 (2008), p. 4719-4729

[2] C. Carmela, and B. L. Hughes, in: Joint channel estimation and data detection in space-time communications, edited by Communications, IEEE chapter, 51.8 (2003), p. 1266-1270.

[3] G.Jingpeng, et al. in: SAGE-ISD joint estimation and detection algorithm in MIMO-OFDM systems, edited by Journal of Central South University (Science and Technology), chapter, 10 (2013), p. 021.

[4] G. Jing-Peng, et al. in: Joint estimation and detection algorithm in MIMO-OFDM systems, edited by Journal of Jilin University (Engineering and Technology Edition), chapter, 44(2014),p. 861-866. 